\section{Prevalência de parasitos intestinais na comunidade indígena Maxakali, Minas Gerais, Brasil, 2009}

\author{
Prevalence of intestinal parasites in the Maxakali \\ indigenous community in Minas Gerais, \\ Brazil, 2009
}

\section{Prevalencia de parásitos intestinales en la comunidad indígena Maxakali, Minas Gerais, Brasil, 2009}

\author{
1 Universidade Vale do Rio \\ Doce, Governador Valadares, \\ Brasil. \\ Coodenação Técnica \\ Operacional Maxakali, \\ Distrito Sanitário Especial \\ Indigena de Minas Gerais/ \\ Espírito Santo, Governador \\ Valadares, Brasil. \\ ${ }^{3}$ Universidade Presidente \\ Antônio Carlos, Teófilo Otoni, \\ Brasil. \\ ${ }_{4}^{4}$ Escola de Engenharia, \\ Universidade Federal de \\ Minas Gerais, Belo Horizonte, \\ Brasil. \\ ${ }^{5}$ Faculty of Epidemiology \\ and Population Health \\ London School of Hygiene \\ and Tropical Medicine, \\ London, $U . K$. \\ ${ }_{6}$ Escola de Medicina \\ Universidade Federal de Ouro \\ Preto, Ouro Preto, Brasil. \\ Correspondência \\ G. L. L. Machado-Coelho \\ Escola de Medicina \\ Universidade Federal de \\ Ouro Preto. \\ Campus Universitário, Ouro \\ Preto, $M G$ \\ 35400-000, Brasil. \\ gmcoelho@medicina.ufop.br
}

\begin{abstract}
A prevalence survey using the TF-Test technique to identify intestinal parasites was conducted in the Maxakali indigenous villages in Minas Gerais State, Brazil. Stool samples were collected on three alternating days, in separate tubes, containing 10\% formalin, and unified in a laboratory by double filtering centrifugation. Samples of sediment aliquot were prepared in triplicate and examined by microscope (10x and 40x) for eggs, cysts, and larvae. Prevalence of parasites (89.5\%) and polyparasitism (46\%) were similar by sex and age, but varied by village. Prevalent species were: Entamoeba histolytica/Entamoeba dispar (48.9\%), Giardia duodenalis (32\%), Entamoeba coli (40.8\%), Endolimax nana (10.3\%), hookworms (37.9\%), Schistosoma mansoni (23.7\%), Hymenolepis nana (18.6\%), Strongyloides stercoralis (5.4\%), Ascaris lumbricoides (4.9\%), and Trichuris trichiura (0.5\%). The Maxakali population lives in socially vulnerable conditions, and government agencies need to introduce measures to improve sanitation infrastructure and health education.

Indigenous Population; Parasitic Diseases; Social Vulnerability
\end{abstract}

Eliseu Miranda de Assis 1

Roberto Carlos de Olivieria 2

Luciano Evangelista Moreira ${ }^{3}$

João Luiz Pena 4

Laura Cunha Rodrigues 5

George Luiz Lins Machado-Coelho 6

\section{Resumo}

O objetivo deste estudo foi determinar a prevalência de parasitos intestinais na etnia Maxakali em Minas Gerais, Brasil. Os exames parasitológico das fezes foram realizados através da técnica TF-Test, sendo as amostras fecais coletadas em três dias alternados, em tubos independentes, contendo formol a 10\%, unificados para dupla filtragem por centrifugação. Triplicatas de uma alíquota do sedimento foram examinadas em microscópio (10x e 40x) para identificação de ovos, cistos e larvas. A prevalência de parasitos (89,5\%) e do poliparasitismo (46\%) foi semelhante quanto ao sexo e idade, $e$ variou entre as aldeias. As espécies prevalentes foram: Entamoeba histolytica/Entamoeba dispar (48,9\%), Giardia duodenalis (32\%), Entamoeba coli (40,8\%), Endolimax nana (10,3\%), ancilostomídeos (37,9\%), Schistosoma mansoni (23,7\%), Hymenolepis nana (18,6\%), Strongyloides stercoralis $(5,4 \%)$, Ascaris lumbricoides (4,9\%) $e$ Trichuris trichiura $(0,5 \%)$. Os Maxakali vivem em condições de vulnerabilidade social, e medidas de infraestrutura e de educação em saúde necessitam ser implementadas pelas instituições governamentais.

População Indígena; Doenças Parasitárias; Vulnerabilidade Social 


\section{Introdução}

As parasitoses intestinais estão amplamente disseminadas entre os povos indígenas brasileiros, em função das condições socioeconômico-culturais a que estão expostos e ainda são um problema de saúde pública negligenciado 1,2. Vários autores $3,4,5,6,7,8,9,10,11$ têm relatado que a precariedade das condições de saneamento, característica marcante das áreas indígenas, associada a um certo grau de sedentarismo 7,11 e a hábitos culturais, alimentares e de higiene pessoal 11, são os principais fatores responsáveis pela alta prevalência das parasitoses intestinais nessas comunidades.
Apesar de Minas Gerais ser o estado brasileiro com a quinta maior população indígena do país, estimada em 2000, em 7.376 indivíduos ${ }^{12}$, pouco se conhece sobre o perfil epidemiológico dos povos indígenas do estado. Os Tikmu'un, nos dias atuais, são reconhecidos pelo Estado brasileiro como Maxakali, um conjunto de povos de cerca de 1.500 pessoas 13 , falantes da língua Maxakali. Vivem em quatro localidades, no extremo nordeste de Minas Gerais (Figura 1), na fronteira com o Estado da Bahia. Nos municípios de Santa Helena de Minas e Bertópolis estão, respectivamente, as terras indígenas (TI) de Água Boa e Pradinho; no Município de Ladainha, a TI Aldeia Verde e, no Distrito Topázio do Município de Teófilo Otoni,

Figura 1

Localização dos pólos-base (PB) Maxakali, região nordeste de Minas Gerais, Brasil.
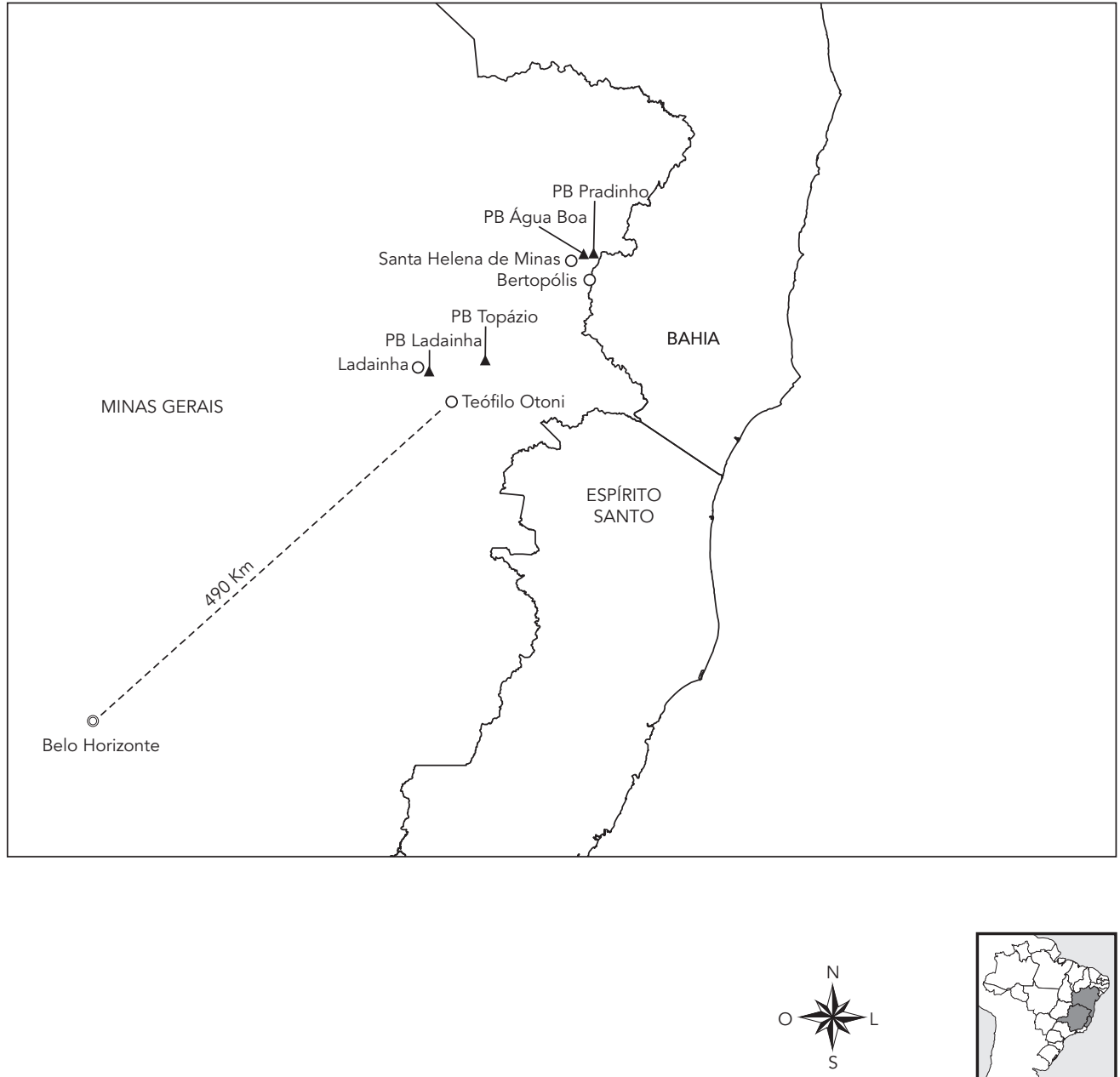
criou-se recentemente a TI Aldeia Cachoeirinha. A soma da extensão destas terras é de aproximadamente 6.434 hectares. Segundo o Sistema de Informação da Atenção à Saúde Indígena (SIASI) para o ano de 2009, a população estava distribuída, conforme sua organização geo-políticosocial, em quatro pólos-base (PB) do tipo I: Água Boa com nove aldeias (Badé, Joviel, Marcelo, Valdemar, Kokiti, Zé Pirão, Maria Diva, Gilmar e Amantchui), Pradinho com três aldeias (Vila Nova, Cachoeira e Maravilha), Ladainha também com três aldeias (Noêmia, Rominho e Gilberto) e Topázio com a aldeia de Rafael.

Os Maxakali, uma das sociedades indígenas originárias das zonas de Mata Atlântica, constituem-se num povo tradicionalmente seminômade, caçador e coletor. Atualmente são um grupo relativamente isolado do ponto de vista cultural e linguístico, pertencente ao tronco Macro-Gê 14 . Seu território histórico estendeu-se além dos Vales do Mucuri e Jequitinhonha, no nordeste de Minas Gerais e sul da Bahia. Mas mesmo após os difíceis séculos de contato com a sociedade envolvente e a perda das terras e de seus recursos naturais, ainda preservam com grande resistência sua língua e práticas simbólicas, mantendo ativa a sua cultura. O português é falado principalmente pelas lideranças e homens, embora, entre si, se comuniquem em seu idioma. As mulheres e crianças, em geral, falam apenas em sua língua nativa, utilizando, quando necessário, palavras esparsas em português.

A população Maxakali apresenta um perfil de morbi-mortalidade com altas taxas de mortalidade especialmente derivada dos altos índices de desnutrição, do consumo frequente de bebidas de alto teor alcoólico, brigas e assassinatos em conflitos internos e nos contatos com a sociedade envolvente, sendo que entre as doenças que mais acometem os índios estão as diarreias, as doenças respiratórias e as escabioses 15.

O nível de exclusão social nessa etnia é muito elevado, quase $85 \%$ das famílias vivem com renda mensal igual ou inferior a meio salário mínimo, que na sua maioria advém dos recursos de programas governamentais, onde apenas $1 / 3$ das famílias estão cadastradas no Programa Bolsa Família e Bolsa Escola. Portanto, frente à vulnerabilidade social da etnia Maxakali, o objetivo desse estudo foi determinar a prevalência das parasitoses intestinais nesse grupo populacional.

\section{Material e métodos}

Um inquérito de prevalência censitário, para avaliar o estado nutricional, a frequência de parasitos intestinais e as condições sanitárias, foi realizado em todas as aldeias Maxakali. Dados do número de habitantes e domicílios foram obtidos por meio da base de dados do Distrito Sanitário Especial Indígena (DSEI) de Minas Gerais/Espírito Santo. A população é jovem e sua composição por sexo e idade, em 2009, está apresentada na Tabela 1. A distribuição dos Maxakali nos quatro PB era: PB Água Boa $(\mathrm{N}=673)$, PB Pradinho ( $\mathrm{N}=619)$, PB Ladainha $(\mathrm{N}=130)$ e PB Topázio $(\mathrm{N}=75)$.

O tipo de construção e as condições sanitárias dos domicílios foram registrados em questionário estruturado e obtidos a partir da observação visual das condições existentes. As variáveis observadas foram tipo de parede, revestimento, cobertura, piso, eletricidade; origem e conservação

Tabela 1

Composição da população Maxakali, por sexo e idade, em 2009.

\begin{tabular}{lccc}
\hline Faixa etária (anos) & Masculino & Sexo & Total (\%) \\
& Feminino & $84(5,6)$ \\
$<1$ & 45 & 39 & $384(25,7)$ \\
$1-5$ & 170 & 214 & $311(20,8)$ \\
$6-12$ & 167 & 144 & $213(14,2)$ \\
$13-18$ & 113 & 100 & $259(17,3)$ \\
$19-29$ & 136 & 123 & $98(6,5)$ \\
$30-39$ & 45 & 53 & $88(5,9)$ \\
$40-49$ & 39 & 49 & $29(1,9)$ \\
$50-59$ & 15 & 14 & $31(2,1)$ \\
$\geq 60$ & 14 & 17 & $1.497(100,0)$ \\
\hline
\end{tabular}


da água de beber e cozinhar; tipo de sanitário; destino dos dejetos e lixo.

Para a realização dos exames parasitológico das fezes foi utilizada a técnica TF-Test (Three Fecal Test) 16, sendo as amostras fecais coletadas em três dias alternados, em tubos independentes, contendo formol $10 \%$, unificados para dupla filtragem por centrifugação. Esse método foi utilizado por apresentar sensibilidade semelhante ao Kato-Katz para a identificação de ovos de helmintos e maior sensibilidade que os métodos de sedimentação para a identificação de cistos de protozoários 16. No laboratório de parasitologia da Universidade Presidente Antônio Carlos (Teófilo Otoni, Minas Gerais) os tubos foram unificados para dupla filtragem por centrifugação. Em cada um dos três tubos com material fecal foi adicionada uma gota de sabão neutro e 1,5mL de acetato de etila para eliminação da gordura. Após centrifugação e obtenção do sedimento, uma alíquota era examinada ao microscópio em triplicata, para visualizar cistos de protozoários (40x) e ovos e larvas de helmintos (10x). A diferenciação entre as larvas de Strongyloides stercoralis e de ancilostomídeos foi realizada pela análise morfológica (40x) das características do vestíbulo bucal e pela presença ou ausência do desenvolvimento do primórdio genital.

O programa SPSS versão 20 (SPSS Inc., Chicago, Estados Unidos) foi utilizado para o processamento e análise estatística dos dados. Foram utilizados os testes do qui-quadrado para comparação das proporções, sendo adotado o nível de 5\% de significância para constatação de diferença estatística. A prevalência foi calculada para uma base de 100 habitantes, e descrita de acordo com o sexo, idade e PB de referência para a saúde.

Esse estudo foi aprovado pelas lideranças indígenas e pelo Comitê de Ética em Pesquisa da Universidade Federal de Ouro Preto (parecer no 2005/58), pela Comissão Nacional de Ética em Pesquisa (CONEP; parecer no 902/2006) e pela Fundação Nacional do Índio (FUNAI; autorização no 73/CGEP/06).

\section{Resultados}

Apesar das lideranças Maxakali terem sido devidamente orientadas quanto à realização da pesquisa, assim como os indígenas em suas residências, durante a observação dos aspectos sanitários e distribuição dos tubos coletores, somente $555(37,1 \%)$ indivíduos entregaram os recipientes para a pesquisa coproparasitológica, mesmo após duas tentativas de coleta. Não houve diferença significativa $(p>0,05)$ na participação quanto ao sexo (feminino: 295/753, masculino: 260/744), mas a participação da faixa etária de 0-12 anos (316/779) foi significativamente maior ( $p=0,003$ ) do que nas outras (13-18 anos: 59/213 19-59 anos: 172/474; 60 anos ou mais: 8/31). A adesão variou significativamente $(\mathrm{p}<0,001)$ também segundo os PB: Ladainha (76,2\%: 99/130), Água Boa (50,2\%: 338/673), Topázio (38,7\%: 29/75) e Pradinho (14,4\%: 89/619). Das amostras recebidas, $146(26,3 \%)$ foram consideradas como perda devido ao pouco material entregue.

Das 409 amostras válidas, isto é, aquelas com três tubos de coleta, $84,3 \%(\mathrm{n}=366)$ estavam positivas para algum tipo de parasita, sem diferença significativa ( $\mathrm{p}>0,05)$ quanto ao sexo [feminino: 88,7\% (189/213); masculino: 90,3\% (177/196)] ou idade, com exceção dos ancilostomídeos (Tabela 2). Todos os pólos-base apresentaram alta prevalência de parasitos intestinais (Tabela 3): PB Ladainha (85,9\%; 85/99), PB Água Boa (88,1\%; 170/193), PB Pradinho (94,3\%; 83/88) e PB Topázio $(96,6 \% ; 28 / 29)$. As espécies prevalentes foram: Entamoeba histolytica/Entamoeba dispar (48,9\%), ancilostomídeos (37,9\%), Giardia duodenalis (32\%), Schistosoma mansoni (23,7\%), Hymenolepis nana (18,6\%), Strongyloides stercoralis (5,4\%), Ascaris lumbricoides (4,9\%) e Trichuris trichiura $(0,5 \%)$. A prevalência de poliparasitismo (indivíduos com dois ou mais destes parasitos) foi elevada (56\%; 229/409), sem diferenças significativas $(\mathrm{p}>0,05)$ quanto ao sexo e idade, mas variando significativamente $(p=0,01)$ quanto ao PB: Água Boa $(39,4 \% ; 76 / 193)$, Ladainha (42,4\%; 42/99), Pradinho (52,3\%, 46/88) e Topázio $(82,8 \%$; 24/29) (Tabela 3). A média de parasitos por indivíduo foi de 2,56 (desvio-padrão $=0,69$ ) (Figura 2). Os protozoários não patogênicos, Entamoeba coli (40,8\%) e Endolimax nana (10,3\%), também apresentaram alta prevalência.

Através da observação in loco dos domicílios Maxakali foi possível constatar as péssimas condições sanitárias onde eles vivem, sendo que dos 166 domicílios avaliados de um total de 175, a maioria residia em casas de palha ou de lona $(83,1 \%)$, piso de terra batida $(92,2 \%)$; sem sanitários $(74,4 \%)$ e sem água tratada $(98,7 \%)$ (Tabela 4).

\section{Discussão}

A prevalência de parasitos intestinais na etnia Maxakali foi alta e em geral semelhante à observada em outras etnias $1,3,4,5,6,7,8,9,10,11$ A alta concentração de indivíduos nos domicílios, o sedentarismo, a convivência direta com animais domésticos, aliados à falta de infraestrutura adequada de abastecimento de água potável 
Tabela 2

Prevalência das parasitos intestinais por idade, em uma amostra da população Maxakali, 2009.

\begin{tabular}{|c|c|c|c|c|c|c|c|c|c|c|c|}
\hline \multirow[t]{4}{*}{ Espécie } & \multicolumn{10}{|c|}{ Faixa etária (anos) } & \multirow[t]{4}{*}{ Valor de $p$ * } \\
\hline & \multirow[t]{2}{*}{$<1$} & \multirow[t]{2}{*}{$1-5$} & \multirow[t]{2}{*}{$6-12$} & \multirow{2}{*}{\multicolumn{4}{|c|}{ 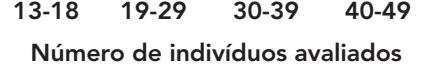 }} & \multirow[t]{2}{*}{$50-59$} & \multirow[t]{2}{*}{$\geq 60$} & \multirow[t]{2}{*}{ Total } & \\
\hline & & & & & & & & & & & \\
\hline & 17 & 112 & 101 & 42 & 74 & 26 & 23 & 10 & 4 & 409 & \\
\hline \multirow[t]{2}{*}{ Entamoeba histolytica/Entamoeba dispar } & 4 & 51 & 49 & 24 & 39 & 14 & 11 & 5 & 3 & 200 & 0,451 \\
\hline & $(23,5)$ & $(45,5)$ & $(48,5)$ & $(57,1)$ & $(52,7)$ & $(53,8)$ & $(47,8)$ & $(50,0)$ & $(75,0)$ & $(48,9)$ & \\
\hline \multirow[t]{2}{*}{ Giardia duodenalis } & 4 & 43 & 28 & 14 & 19 & 9 & 11 & 2 & 1 & 131 & 0,397 \\
\hline & $(23,5)$ & $(38,4)$ & $(27,7)$ & $(33,3)$ & $(25,7)$ & $(34,6)$ & $(47,8)$ & $(20,0)$ & $(25,0)$ & $(32,0)$ & \\
\hline \multirow[t]{2}{*}{ Entamoeba coli } & 4 & 40 & 47 & 17 & 32 & 9 & 13 & 3 & 2 & 167 & 0,410 \\
\hline & $(23,5)$ & $(35,7)$ & $(46,5)$ & $(40,5)$ & $(43,2)$ & $(34,6)$ & $(56,5)$ & $(30,0)$ & $(50,0)$ & $(40,8)$ & \\
\hline \multirow[t]{2}{*}{ Endolimax nana } & 0 & 10 & 7 & 4 & 13 & 3 & 2 & 3 & 0 & 42 & 0,131 \\
\hline & $(0,0)$ & $(8,9)$ & $(6,9)$ & $(9,5)$ & $(17,6)$ & $(11,5)$ & $(8,7)$ & $(30,0)$ & $(0,0)$ & $(10,3)$ & \\
\hline \multirow[t]{2}{*}{ Hymenolepis nana } & 3 & 27 & 18 & 8 & 9 & 8 & 3 & 0 & 0 & 76 & 0,229 \\
\hline & $(17,6)$ & $(24,1)$ & $(17,8)$ & $(19,0)$ & $(12,2)$ & $(30,8)$ & $(13,0)$ & $(0,0)$ & $(0,0)$ & $(18,6)$ & \\
\hline \multirow[t]{2}{*}{ Schistosoma mansoni } & 2 & 21 & 33 & 15 & 13 & 6 & 6 & 1 & 0 & 97 & 0,067 \\
\hline & $(11,8)$ & $(18,8)$ & $(32,7)$ & $(35,7)$ & $(17,6)$ & $(23,1)$ & $(26,1)$ & $(10,0)$ & $(0,0)$ & $(23,7)$ & \\
\hline \multirow{2}{*}{ Ascaris lumbricoides } & 1 & 8 & 5 & 1 & 3 & 0 & 1 & 1 & 0 & 20 & 0,852 \\
\hline & $(5,9)$ & $(7,1)$ & $(5,0)$ & $(2,4)$ & $(4,1)$ & $(0,0)$ & $(4,3)$ & $(10,0)$ & $(0,0)$ & $(4,9)$ & \\
\hline \multirow[t]{2}{*}{ Ancilostomídeos } & 4 & 40 & 45 & 23 & 21 & 13 & 7 & 1 & 1 & 155 & 0,027 \\
\hline & $(23,5)$ & $(35,7)$ & $(44,6)$ & $(54,8)$ & $(28,4)$ & $(50,0)$ & $(30,4)$ & $(10,0)$ & $(25,0)$ & $(37,9)$ & \\
\hline \multirow[t]{2}{*}{ Strongyloides stercoralis } & 2 & 5 & 5 & 5 & 2 & 1 & 1 & 0 & 1 & 22 & 0,272 \\
\hline & $(11,8)$ & $(4,5)$ & $(5,0)$ & $(11,9)$ & $(2,7)$ & $(3,8)$ & $(4,3)$ & $(0,0)$ & $(25,0)$ & $(5,4)$ & \\
\hline \multirow[t]{2}{*}{ Trichuris trichiura } & 0 & 1 & 0 & 0 & 0 & 1 & 0 & 0 & 0 & 2 & 0,460 \\
\hline & $(0,0)$ & $(0,9)$ & $(0,0)$ & $(0,0)$ & $(0,0)$ & $(3,8)$ & $(0,0)$ & $(0,0)$ & $(0,0)$ & $(0,5)$ & \\
\hline
\end{tabular}

* Diferença significativa $(p<0,05)$.

Tabela 3

Prevalência dos parasitos intestinais por pólo-base, em uma amostra da população Maxakali, 2009.

\begin{tabular}{|c|c|c|c|c|c|c|}
\hline \multirow[t]{2}{*}{ Espécie } & \multicolumn{4}{|c|}{ Pólo-base } & \multirow{2}{*}{$\begin{array}{c}\text { Total } \\
\text { (N = 409) } \\
\text { n (\%) }\end{array}$} & \multirow[t]{2}{*}{ Valor de $p$ * } \\
\hline & $\begin{array}{l}\text { Ladainha } \\
(\mathrm{N}=99) \\
\mathrm{n}(\%)\end{array}$ & $\begin{array}{c}\text { Água Boa } \\
(\mathrm{N}=193) \\
\text { n (\%) }\end{array}$ & $\begin{array}{c}\text { Pradinho } \\
(\mathrm{N}=88) \\
n(\%)\end{array}$ & $\begin{array}{c}\text { Topázio } \\
(N=29) \\
\text { n (\%) }\end{array}$ & & \\
\hline 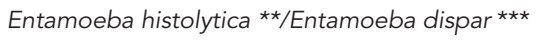 & $48(48,5)$ & $86(44,6)$ & $53(60,2)$ & $13(44,8)$ & $200(48,9)$ & 0,103 \\
\hline Giardia duodenalis ** & $14(14,1)$ & $62(32,1)$ & $43(48,9)$ & $12(41,4)$ & $131(32,0)$ & $<0,001$ \\
\hline Entamoeba coli $\star \star \star ~$ & $30(30,3)$ & $80(41,5)$ & $48(54,5)$ & $9(31,0)$ & $167(40,8)$ & 0,006 \\
\hline 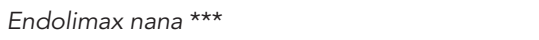 & $14(14,1)$ & $23(11,9)$ & $5(5,7)$ & $0(0,0)$ & $42(10,3)$ & 0,057 \\
\hline Hymenolepis nana ** & $19(19,2)$ & $41(21,2)$ & $9(10,2)$ & $7(24,1)$ & $76(18,6)$ & 0,134 \\
\hline Schistosoma mansoni ** & $32(32,3)$ & $34(17,6)$ & $21(23,9)$ & $10(34,5)$ & $97(23,7)$ & 0,020 \\
\hline Ascaris lumbricoides ** & $2(2,0)$ & $2(1,0)$ & $2(2,3)$ & $14(48,3)$ & $20(4,9)$ & $<0,001$ \\
\hline Ancilostomídeos ** & $30(30,3)$ & $58(30,1)$ & $53(60,2)$ & $14(48,3)$ & $155(37,9)$ & $<0,001$ \\
\hline Strongyloides stercoralis ** & $6(6,1)$ & $13(6,7)$ & $2(2,3)$ & $1(3,4)$ & $22(5,4)$ & 0,446 \\
\hline Trichuris trichiura ** & $0(0,0)$ & $0(0,0)$ & $2(2,3)$ & $0(0,0)$ & $2(0,5)$ & 0,062 \\
\hline Monoparasitismo ** & $33(33,3)$ & $70(36,3)$ & $30(34,1)$ & $4(13,8)$ & $137(33,5)$ & 0,005 \\
\hline Poliparasitismo ** & $42(42,4)$ & $76(39,4)$ & $46(52,3)$ & $24(82,8)$ & $188(46,0)$ & \\
\hline
\end{tabular}

* Diferença significativa $(p<0,05)$;

** Espécies patogênicas;

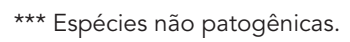




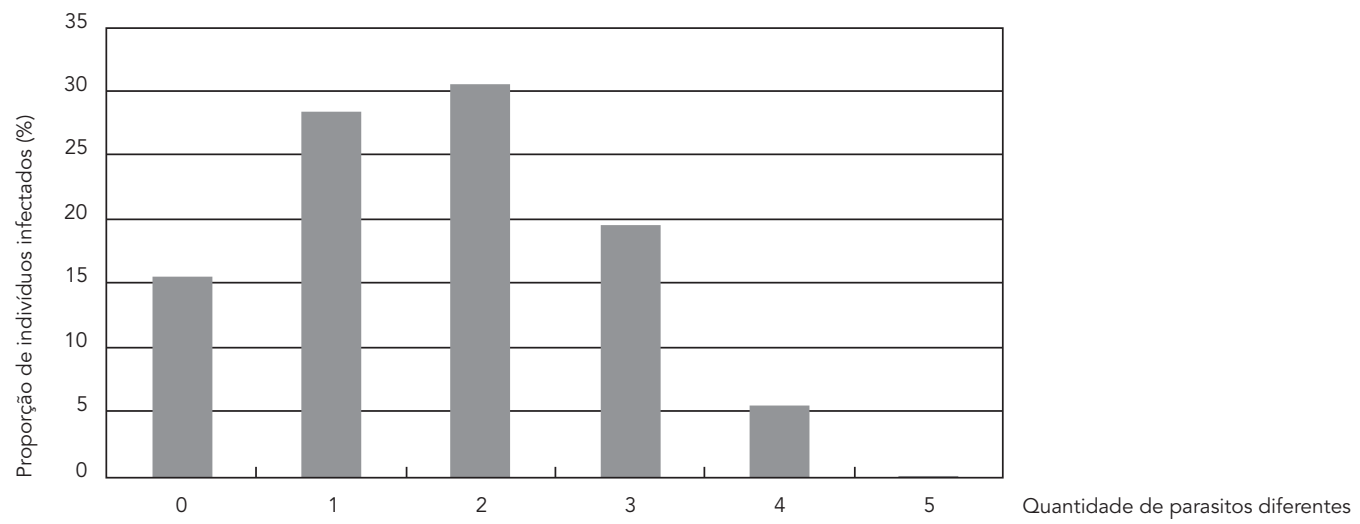

e coleta de dejetos são fatores ambientais que propiciam a propagação de enteropatógenos de veiculação feco-oral 17,18. Nas terras indígenas Maxakali um fator agravante, além da alta concentração de fezes expostas no peridomicílio, é o hábito generalizado de preparar as refeições em uma única panela em fogo do lado de fora das casas junto ao chão, onde as crianças circulam livremente ao redor das casas, e não ser frequente o hábito de lavar as mãos e alimentos, facilitando a contaminação.

Ao contrário do observado pela maioria dos autores, os protozoários patogênicos $E$. histolytica/E. dispar e G. duodenalis foram as espécies mais prevalentes, sendo concordante apenas com o observado por Genaro \& Ferraroni 4 na etnia Nadëb-Maku, por Miranda et al. 6 na etnia Parakanã e por Fontbonne et al. 7 na etnia Pankararu. A diferença entre as prevalências observadas entre as etnias pode ser devida às diferentes técnicas utilizadas. Ao contrário das técnicas de sedimentação espontânea comumente utilizadas em inquéritos coprológicos, neste estudo utilizamos uma técnica que associa centrifugação, filtração e sedimentação e que apresenta alta sensibilidade na detecção de cistos de protozoários 16 . Na medida em que esses protozoários lesam a mucosa intestinal, provocando além da dor abdominal, diarreia e má-absorção, não deveriam ser negligenciados, devido não só ao risco de formas invasivas intestinais e extraintestinais de amebíase 7, mas também frente ao risco de piorar o quadro nutricional dessa população indígena, que convive com o flagelo da desnutrição. Esse quadro se agrava naqueles coinfectados com $G$. duodenalis, um dos principais agentes responsáveis pela diarreia aguda, que pode se cronificar levando à má absorção. O controle dessa protozoose é difícil pois o principal mecanismo de transmissão é o contato pessoa a pessoa, além das vias hídrica e alimentar 19.

As infecções helmínticas também são facilitadoras dos quadros de desnutrição, por dois processos: a fisiopatologia do parasita no trato gastrointestinal e a ingesta reduzida de alimentos 20 , geralmente associada às infecções por $A$. lumbricoides e T. trichiura. Mas ao contrário do esperado, baseado nos achados de outras etnias $1,6,7,9$, a prevalência dessas espécies foi muito baixa, sendo no entanto concordante com o observado na etnia Suruí 21 . Na medida em que não há relato de tratamento em massa nessa população nos últimos anos e considerando as condições propícias para a sua transmissão, novos estudos são necessários para esclarecer esses achados.

Chama atenção, entre os Maxakali, a alta prevalência da ancilostomíase que desempenha um papel deletério importante no quadro nutricional pela anemia causada pela perda de sangue intestinal 22 , e pela deficiência de vitamina A e $\mathrm{C} 23$. As condições ambientais em que vivem os Maxakali, solo arenoso e úmido, e a manutenção do hábito de andar descalços, favorecem respectivamente o desenvolvimento e a penetração das larvas infectantes 20 . Prevalências elevadas para esse parasito também foram observadas em outras etnias indígenas 3,8 .

Apesar da ausência de caramujos do gênero Biomphalaria nas coleções de água naturais dentro das terras indígenas Maxakali (dado não 
Tabela 4

Condições de habitação de 166 dos 175 domicílios das aldeias Maxakali, 2009.

\begin{tabular}{|c|c|c|}
\hline Condições de habitação & n & $\%$ \\
\hline \multicolumn{3}{|l|}{ Tipo de casa } \\
\hline \multicolumn{3}{|l|}{ Parede } \\
\hline Palha/Lona & 138 & 83,1 \\
\hline Outro & 28 & 16,9 \\
\hline \multicolumn{3}{|l|}{ Cobertura } \\
\hline Palha/Sapé/Lona & 80 & 48,2 \\
\hline Telha de barro & 86 & 51,8 \\
\hline \multicolumn{3}{|l|}{ Piso } \\
\hline Terra batida & 153 & 92,2 \\
\hline \multicolumn{3}{|l|}{ Energia elétrica } \\
\hline Sem energia & 35 & 21,0 \\
\hline \multicolumn{3}{|l|}{ Número de cômodos } \\
\hline $1-2$ & 149 & 89,8 \\
\hline 3 ou + & 17 & 10,2 \\
\hline \multicolumn{3}{|l|}{ Condições sanitárias } \\
\hline \multicolumn{3}{|l|}{ Abastecimento de água } \\
\hline Apenas do poço artesiano & 35 & 21,3 \\
\hline Minas de água & 8 & 4,9 \\
\hline Rios/Lagoas/Outros & 123 & 73,8 \\
\hline Sem tratamento da água & 150 & 98,7 \\
\hline \multicolumn{3}{|l|}{ Sistema de esgoto sanitário } \\
\hline Ausência de vaso sanitário & 124 & 74,7 \\
\hline Céu aberto/Rio & 26 & 15,7 \\
\hline Fossa séptica & 16 & 9,6 \\
\hline \multicolumn{3}{|l|}{ Destino do lixo } \\
\hline Queimado/Enterrado & 60 & 36.5 \\
\hline Exposto & 106 & 63,5 \\
\hline Total de domicílios & 166 & 100,0 \\
\hline
\end{tabular}

mostrado), foi interessante observar a presença de vários indivíduos infectados pelo S. mansoni. Esses dados apontam para a necessidade de identificar a fonte de infecção dessas comunidades. Apesar dos hábitos seminômandes atuarem como fator protetor para as infecções intestinais 9 , a frequente mobilidade familiar dos Maxakali dentro da região endêmica 24 nos vales dos rios Mucuri e Doce, nas vias de acesso que ligam a região nordeste de Minas Gerais às cidades de Teófilo Otoni e Governador Valadares, poderia explicar em parte a prevalência desse parasito.

De forma inesperada, foi detectada uma alta prevalência da infecção por $H$. nana. Apesar de a transmissão se processar de pessoa a pessoa, geralmente sem a presença de hospedeiro intermediário, através da ingestão de ovos eliminados nas fezes 25 , faz-se necessário analisar os cereais distribuídos e estocados. Pois estes quando mal conservados costumam ficar infestados por coleópteros do gênero Tenebrio, que ao conterem larvas cisticercóides do H. nana, podem causar hiperinfecção quando ingeridos. No entanto, a importância destes, segundo vários autores, é discutível, considerando que o homem parasitado é a principal e a mais importante fonte de contaminação 25.

A alta proporção de indivíduos poliparasitados por espécies patogênicas nessa população também tem sido observada em outras etnias 7,8,9. Fatores que poderiam explicar em parte $o$ poliparasitismo frequente são: alta concentração de pessoas nas aldeias e nas residências, poucos sanitários (os existentes nessa comunidade são de uso coletivo, utilizados mais frequentemente nos festejos nas aldeias), casas de taipa sem revestimento ou de lona, de piso de terra batida, falta de tratamento da água de consumo, recipientes 
de acondicionamento da água improvisados em baldes, panelas, etc., sem a devida higiene, não sendo comum a lavagem das mãos e dos alimentos. Esse quadro se assemelha ao observado por Jensen et al. 26, em comunidades rurais pobres, que mesmo quando a água é obtida de uma fonte segura, esta pode tornar-se contaminada durante a estocagem nas casas. As más condições sanitárias e de higiene dos Maxakali também ficam constatadas pela alta frequência de infecção por protozoários não patogênicos (E. coli e E. nana). A contaminação fecal da água de consumo e alimentos tem exposto essa população, principalmente a infantil, a frequentes surtos epidêmicos de diarreia, responsáveis diretas pela maioria dos óbitos na população de 0 a 5 anos.

Os efeitos do poliparasitismo não são bem conhecidos, mas na infância as múltiplas infecções por diferentes espécies de helmintos podem ter um impacto negativo na nutrição do hospedeiro através de vários mecanismos incluindo anorexia, perda de sangue crônica e má absorção influenciando diretamente a capacidade cognitiva e o desempenho motor, além de influir significativamente no processo de crescimento em crianças que vivem em áreas endêmicas. Embora as consequências nutricionais estejam mais relacionadas a maiores cargas parasitárias, populações vulneráveis mesmo com cargas parasitárias menores podem apresentar déficits de crescimento e nutricionais ${ }^{20}$. Repercutindo sobre o desenvolvimento cognitivo, o parasitismo pode consequentemente manter a condição de vulnerabilidade social do grupo, o baixo nível de instrução e a incapacidade laboral, para as atividades ligadas à prática e aprendizado da própria cultura indígena 27.

A distribuição das espécies de parasitos por faixa etária, apesar de não significativa, exceto para os ancilostomídeos, seguiu o padrão geralmente observado por outros autores 28 . A. lumbricoides, apesar de pouco prevalente, predominou naqueles com menos de 12 anos (14/20); $S$. mansoni na faixa etária entre 6 e 29 anos (61/97), mas também com número importante entre aqueles entre 1 e 5 anos (21/97); ancilostomídeos (142/155) entre 1 e 39 anos; H. nana (73/76) até 40 anos; e E. histolytica/E. dispar e G. duodenalis reduzindo o número de casos em função da idade.

A principal limitação deste estudo foi a baixa adesão $(36,6 \%)$ na entrega dos frascos com amostras de fezes. Foi perceptível que, de acordo com o engajamento das lideranças locais, a frequência de participação por parte da aldeia era maior. Outra limitação foi a possível subestimação da prevalência de $S$. stercoralis e Enterobius vermicularis, pois o método empregado apresenta baixa sensibilidade na identificação desses parasitos. Entretanto, a partir desses resultados, as equipes de saúde local, pela primeira vez, realizaram tratamento em massa da população Maxakali, com anti-helmintos e antiprotozoários não sistêmicos. Todos os indivíduos com esquistossomose receberam o tratamento específico recomendado pelo Ministério da Saúde, e estão sendo acompanhados pela equipe médica local e por nossa equipe.

\section{Conclusão}

Esses resultados apontam para precárias condições de saneamento das aldeias Maxakali, que resultam em altos níveis de parasitismo intestinais, e indicam a necessidade de implementação, pelas autoridades responsáveis, de medidas urgentes de saneamento básico, programas de educação em saúde e tratamento em massa periódico, contando com a efetiva participação dos Maxakali. No entanto, os desafios para a questão indígena não se resumem às doenças ou ausência de saneamento, mas a uma realidade mais ampla e abrangente que coloca os povos indígenas numa condição de não conformidade em relação às políticas públicas destinadas aos não indígenas, em todos os campos. 


\section{Resumen}

El objetivo de este estudio fue determinar la prevalencia de parásitos intestinales en la etnia Maxakali. Los exámenes parasitológicos de las heces se realizaron a través de la técnica TF-Test, las muestras fecales se recogieron en tres días alternados, en tubos independientes, conteniendo formol a un 10\%, unificados para un doble filtrado por centrifugación. Triplicados de una alícuota del sedimento se examinaron en microscopio (10x y 40x) para la identificación de huevos, quistes y larvas. La prevalencia de parásitos (89,5\%) y del poliparasitismo (46\%) fue semejante en cuanto al sexo y edad, y varió entre las aldeas. Las especies prevalentes fueron: Entamoeba histolytica/Entamoeba dispar $(48,9 \%)$, Giardia duodenalis (32\%), Entamoeba coli (40,8\%), Endolimax nana (10,3\%), ancilostomídeos (37,9\%), Schistosoma mansoni (23,7\%), Hymenolepis nana $(18,6 \%)$, Strongyloides stercoralis $(5,4 \%)$, Ascaris lumbricoides $(4,9 \%) y$ Trichuris trichiura $(0,5 \%)$. Los Maxakali viven en condiciones de vulnerabilidad social, y medidas de infraestructura y de educación en salud necesitan ser implementadas por instituciones gubernamentales.

Población Indígena; Enfermedades Parasitarias; Vulnerabilidad Social

\section{Referências}

1. Santos RV, Coimbra Jr. CEA, Ott AMT. Estudos epidemiológicos entre grupos indígenas de Rondônia. III. Parasitoses intestinais nas populações dos vales dos rios Guaporé e Mamoré. Cad Saúde Pública 1985; 1:467-77.

2. Coimbra Jr. CEA, Santos RV. Perfil epidemiológico da população indígena no Brasil. Porto Velho: Universidade Federal de Rondônia/Rio de Janeiro: Escola Nacional de Saúde Pública, Fundação Oswaldo Cruz; 2001. (Documento de Trabalho, 3).

3. Coimbra Jr. CEA, Mello DA. Enteroparasitas e $\mathrm{Ca}$ pillaria sp. entre o grupo Suruí, Parque Indígena Aripuanã, Rondônia. Mem Inst Oswaldo Cruz 1981; 76:299-302.

4. Genaro O, Ferraroni JJ. Estudo sobre malária e parasitoses intestinais em indígenas da tribo NadebMaku, Estado do Amazonas, Brasil. Rev Saúde Pública 1984; 18:162-9.

5. Coimbra Jr. CEA, Santos RV. Parasitismo intestinal entre o grupo indígena Zoró, Estado de Mato Grosso (Brasil). Cad Saúde Pública 1991; 7:100-3.

\section{Colaboradores}

E. M. Assis participou da coleta, processamento e análise dos dados e da redação do artigo. R. C. Oliveira colaborou na coordenação das atividades de campo, na coleta de dados e auxiliou na revisão do artigo. L. E. Moreira realizou a análise parasitológica e auxiliou na revisão do artigo. J. L. Pena participou da coleta de dados e na revisão do artigo. L. C. Rodrigues fez a revisão geral e aprovou a versão final. G. L. L. Machado-Coelho definiu o desenho epidemiológico, realizou a análise estatística e redigiu o artigo.

\section{Agradecimentos}

Agradecemos à equipe do Distrito Sanitário Especial Indígena de Minas Gerais/Espírito Santo e ao setor de transporte da Universidade Vale do Rio Doce pelo apoio logístico para a realização desse trabalho. À Fundação de Amparo à Pesquisa de Minas Gerais (FAPEMIG; APQ 3200-4.01/07; APQ 01161-10) e Conselho Nacional de Desenvolvimento Científico e Tecnológico (CNPq; 402530/2008-0; 302315/2011-0) pelo financiamento.
6. Miranda RA, Xavier FB, Menezes RC. Parasitismo intestinal em uma aldeia indígena Parakanã, sudeste do Estado do Pará, Brasil. Cad Saúde Pública 1998; 14:507-11.

7. Fontbonne A, Freese-de-Carvalho EF, Acioli MD, Sá GA, Cesse EAP. Fatores de risco para poliparasitismo intestinal em uma comunidade indígena de Pernambuco, Brasil. Cad Saúde Pública 2001; 17:367-73.

8. Gillio J, Mioranza SL, Takizawa MGMH. Parasitismo intestinal em índios da reserva indígena Rio das Cobras, Cascável, Paraná. Rev Bras Anal Clín 2006; 38:193-5.

9. Rios L, Cutolo SA, Giatti LL, Castro M, Rocha AA, Toledo RF, et al. Prevalência de parasitos intestinais e aspectos socioambientais em comunidade indígena no distrito de Iauaretê, município de São Gabriel da Cachoeira (AM), Brasil. Saúde Soc 2007; 16:76-86. 
10. Bóia MN, Carvalho-Costa FA, Sodré FC, Porras-Pedroza BE, Faria EC, Magalhães GAP, et al. Tuberculosis and intestinal parasitism among indigenous people in the Brazilian Amazon region. Rev Saúde Pública 2009; 43:176-8.

11. Escobar-Pardo ML, Godoy APOG, Machado RS, Rodrigues D, Fagundes Neto U, Kawakami E. Prevalência de parasitoses intestinais em crianças do Parque Indígena do Xingu. J Pediatr (Rio J.) 2010; 86:493-6.

12. Dias Júnior CS, Verona APA, Pena JL, MachadoCoelho GLL. Crescimento da população indígena em Minas Gerais: análise da influência da dinâmica demográfica e reclassificação racial a partir dos dados censitários de 1991-2000. Rev Bras Promoç Saúde 2009; 22:157-63

13. Alvarenga ACS. Música na cosmologia Maxakali. Um olhar sobre um ritual do Xũnĩm - uma par titura sonoro-mítico-visual [Dissertação de Mestrado]. Belo Horizonte: Universidade Federal de Minas Gerais; 2007.

14. Tugny RP. Cantobrilho Tikmu'um: nos limites do país fértil. Rio de Janeiro: Museu do Índio; 2010.

15. Las Casas R. Saúde Maxakali, recursos de cura e gênero: análise de uma situação social [Dissertação de Mestrado]. Rio de Janeiro: Universidade do Estado do Rio de Janeiro; 2007.

16. Carvalho GLX, Moreira LE, Pena JL, Marinho CC, Bahia MT, Machado-Coelho GLL. A comparative study of the TF-Test, Kato-Katz, Hoffman-PonsJaner, Willis and Baermann-Moraes coprologic methods for the detection of human parasitosis. Mem Inst Oswaldo Cruz 2012; 107:80-4.

17. Linhares AC. Epidemiologia das infecções diarréicas entre populações indígenas da Amazônia. Cad Saúde Pública 1992; 8:121-8.

18. Santos RV, Linhares CA, Coimbra Jr. CEA. Estudos epidemiológicos entre grupos indígenas de Rondônia. IV. Inquérito sorológico para rotavírus entre Suruí e Karitiána. Rev Saúde Pública 1991; 25:230-2.

19. Esrey SA, Collett J, Miuotis MD, Koornhof HJ, Makhale P. The risk of infection from Giardia lamblia due to drinking water supply, use of water and latrines among preschool children in rural Lesotho. Int J Epidemiol 1989; 18:248-53.
20. Crompton DWT, Nesheim MC. Nutritional impact of intestinal helminthiasis during the human life cycle. Annu Rev Nutr 2002; 22:35-59.

21. Coimbra Jr. CEA, Santos RV. Avaliação do estado nutricional num contexto de mudança sócio-econômica: o grupo indígena Suruí do Estado de Rondônia, Brasil. Cad Saúde Pública 1991; 7:538-62.

22. Hotez PJ, Bundy DAP, Beegle K, Brooker S, Drake L, Silva N, et al. Helminth infections: soil-transmitted helminth infections and schistosomiasis. In: Jamison DT, Breman JG, Measham AR, Alleyne G, Claeson M, Evans DB, et al., editors. Disease control priorities in developing countries. 2nd Ed. Washington DC: World Bank; 2006. p. 467-82.

23. Hlaing T. Ascariasis and childhood malnutrition Parasitology 1993; 107 Suppl:S125-36.

24. Souza CP, Caldeira RL, Drummond SC, Melo AL, Guimarães CT, Soares DM, et al. Geographical distribution of Biomphalaria snails in the State of Minas Gerais, Brazil. Mem Inst Oswaldo Cruz 2001; 96:293-302.

25. Rocha RS, Carvalho OS, Santos JS, Katz N. Tentativa de controle de Hymenolepis nana através de tratamentos clínicos repetidos, com praziquantel, em uma comunidade fechada. Rev Saúde Pública $1981 ; 15: 364-70$

26. Jensen PK, Ensink JHJ, Jayasinghe G, van der Hoek W, Cairncross S, Dalsgaard A. Domestic transmission routes of pathogens: the problem of in-house contamination of drinking water during storage in developing countries. Trop Med Int Health 2002; 7:604-9.

27. World Health Organization. Preventive chemotherapy in human helminthiasis: coordinated use of anthelminthic drugs in control interventions: a manual for health professionals and programme managers. Geneva: World Health Organization; 2006.

28. Fleming FM, Brooker S, Geiger SM, Caldas IR, Correa-Oliveira R, Hotez PJ, et al. Synergistic associations between hookworm and other helminth species in a rural community in Brazil. Trop Med Int Health 2006; 11:56-64.

Recebido em 08/Jul/2012

Versão final reapresentada em 07/Out/2012 Aprovado em 30/Nov/2012 